\title{
A SUBSPACE-BASED APPROACH TO SEA CLUTTER SUPPRESSION FOR IMPROVED TARGET DETECTION
}

\author{
Sandeep P. Sira, Douglas Cochran, Antonia Papandreou-Suppappola \\ Darryl Morrell ${ }^{\dagger}$, William Moran ${ }^{\ddagger}$, Stephen Howard ${ }^{\S}$ \\ SenSIP Center, Dept. of Electrical Engineering, Arizona State University \\ $\dagger$ Dept. of Engineering, Arizona State University at the Polytechnic Campus \\ ${ }^{\ddagger}$ Dept. of Electrical Engineering, University of Melbourne \\ $\S$ Defence Science and Technology Organisation, Australia
}

\begin{abstract}
A key issue in detection of small targets on the ocean surface using active radar is low signal-to-clutter ratio (SCR), particularly in situations involving low grazing angle and high sea state. When sufficiently high pulse repetition rates are available, it is possible to obtain several samples of the clutter in a time interval short enough that key contributors to the clutter remain approximately stationary. This paper develops an approach to estimate the clutter subspace in such scenarios and exploit this estimate for clutter suppression and improved detection performance.
\end{abstract}

\section{INTRODUCTION}

The detection of small targets on the ocean surface remains a challenging problem due to the low SCR. Further, sea clutter returns exhibit significant spatial and temporal correlations which must be incorporated into detection strategies. We present a subspace-based approach that exploits the difference in the statistics of the returns from sea clutter and the target to improve detection performance. A detector based on sea clutter statistics is developed. To exploit recent advances in sensing technologies that permit the dynamic adaptation of waveforms on-the-fly, we provide an extension of the approach to waveform-agile detection.

Early studies of detection and waveform design for sea clutter rejection typically assumed a Gaussian model for sea clutter echoes [1]. With modern radars however, the resolution is high enough to resolve the small-scale structure of the sea surface. In this scenario, the Gaussian model, which derives from the application of the central limit theorem to a large number of independent scattering centers, is no longer appropriate and cannot account for the increased presence of spikes in the returns. This has motivated the compoundGaussian (CG) model for sea clutter [2].

This work was supported by the DARPA Waveforms for Active Sensing (WAS) Program under NRL grant N00173-06-1-G006.
In this model, sea clutter returns are believed to be the result of two processes. The first process is called speckle, and is the result of reflections of the incident beam by multiple independent scattering centers. The second process, termed texture, is caused by the large-scale structures of the sea such as swell and wind, and modulates the local mean power of the speckle. The $\mathrm{CG}$ model has received empirical as well as theoretical support $[3,4]$. It has long been known that sea clutter echoes exhibit temporal and spatial correlation [5]. In particular, the speckle decorrelates rapidly (1-5 ms), while the texture remains correlated over several seconds. In practice, the correlation properties of the clutter are determined by the speckle component [4].

In this paper, we propose a method to improve target detection performance in heavy sea clutter. Under the assumption that the radar scene remains practically stationary over several snapshots, we demonstrate that the radar returns from the ocean surface, when imaged at a high pulse repetition frequency (PRF), can provide a waveform-independent estimate of the subspace occupied by the clutter. The orthogonal projection of the received signal on this subspace provides a clutter-suppressed signal. A similar approach to improve detection performance was used in [6], where the subspace of the sea clutter returns was estimated using a Karhunen-Loève decomposition. We estimate the value of the texture in each range bin and use it to form a generalized likelihood ratio test (GLRT) detector. We demonstrate using simulations based on real clutter data that this approach yields significant improvement in detection performance.

The paper is organized as follows. In Section 2, we describe the CG model in detail, while Section 3 develops the received signal model. In Section 4, we present the subspace estimation and clutter suppression algorithms, and in Section 5 , we describe the GLRT detector. Simulation results are presented in Section 6. 


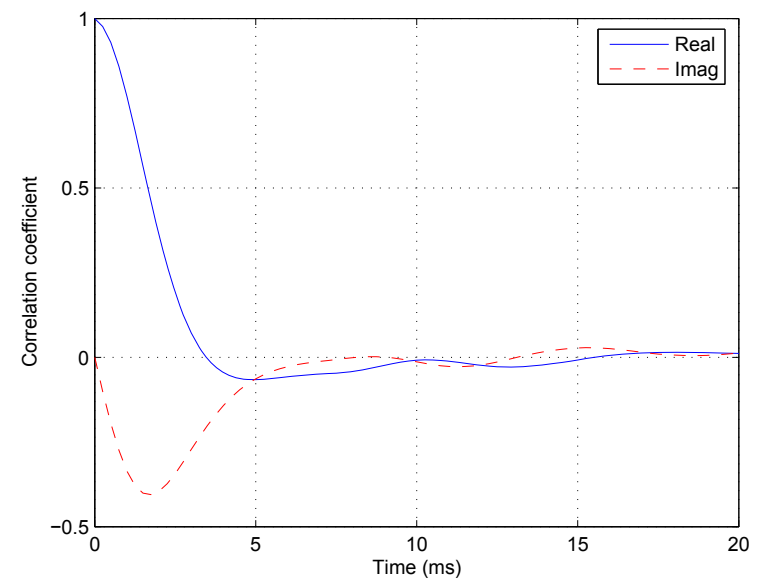

Fig. 1. Correlation coefficient of the speckle component.

\section{COMPOUND-GAUSSIAN CLUTTER MODEL}

In this section, we present a mathematical model for sea clutter returns [5]. In particular, we describe the correlation properties derived from real sea clutter data.

Let $\mathbf{x}_{i}=\left[x_{i}^{0}, x_{i}^{1}, \ldots, x_{i}^{K-1}\right]^{T}$ represent the complex reflectivity of the $i$ th clutter scatterer when it is illuminated with $K$ pulses of a radar signal. The CG model states that $\mathbf{x}_{i}$ follows a complex Gaussian probability distribution with zero mean and covariance matrix $\mathcal{T}_{i} \boldsymbol{\Sigma}$, where $\mathcal{T}_{i} \geq 0$ is the texture, and $\boldsymbol{\Sigma} \in \mathbb{C}^{K \times K} \neq \mathbb{I}_{K}$ is the speckle covariance matrix with $\mathbb{I}_{K}$ denoting the $K \times K$ identity matrix [7]. Given the texture and the speckle covariance matrix, the reflectivities of two scatterers $\mathbf{x}_{i}$ and $\mathbf{x}_{j}$ are independent so that

$$
p\left(\mathbf{x}_{i}, \mathbf{x}_{j} \mid \mathcal{T}_{i}, \mathcal{T}_{j}, \boldsymbol{\Sigma}\right)=p\left(\mathbf{x}_{i} \mid \mathcal{T}_{i}, \boldsymbol{\Sigma}\right) p\left(\mathbf{x}_{j} \mid \mathcal{T}_{j}, \boldsymbol{\Sigma}\right),
$$

where $p(a \mid b)$ is the probability density function of $a$ given $b$.

The correlation properties of the speckle and texture are shown in Fig. 1 and Fig. 2, respectively. These have been obtained by analyzing real sea clutter data, collected at the Osborne Head Gunnery Range (OHGR) with the McMaster University IPIX radar [8]. In particular, we note that the speckle decorrelates within a few milliseconds while the texture decorrelates much more slowly.

\section{RECEIVED SIGNAL MODEL}

We consider a radar operating at a PRF $>10 \mathrm{kHz}$ so that $K=10$ pulses can be transmitted in each dwell within a duration of $1 \mathrm{~ms}$, during which we assume that the radar scene stays practically stationary. This implies that the number of scatterers, their delays and Doppler shifts remain constant during a dwell. In addition, the clutter returns in a dwell will be correlated according to the specular correlation shown in Fig. 1, while the texture can be assumed to be constant.

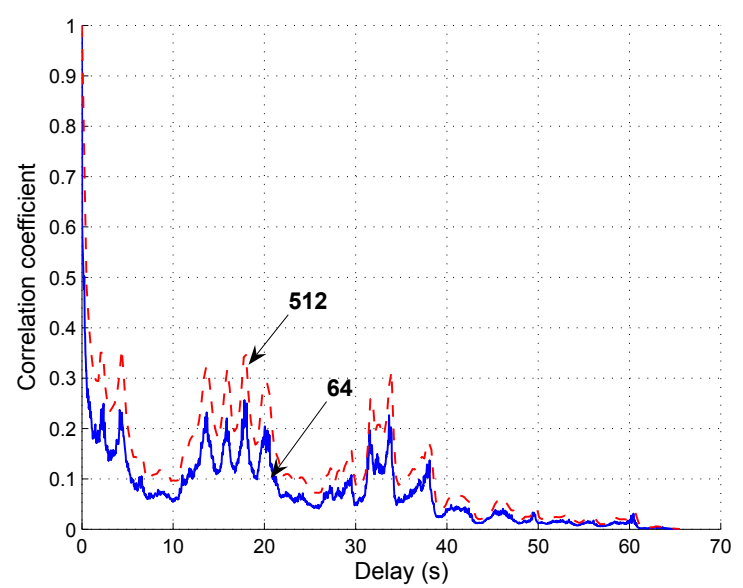

Fig. 2. Correlation coefficient of the texture component. The figures indicate the number of samples that are averaged to obtain each texture estimate.

If $s(t)$ is the signal transmitted in each pulse, the received signal in the $k$ th pulse, $k=0,1, \ldots, K-1$, is given by

$g^{k}(t)=b^{k} s\left(t-\tau_{0}\right) \mathrm{e}^{j 2 \pi \nu_{0} t}+\sum_{i} x_{i}^{k} s\left(t-\tau_{i}\right) \mathrm{e}^{j 2 \pi \nu_{i} t}+n(t)$,

where $b^{k}, \tau_{0}$ and $\nu_{0}$ are the complex reflectivity, delay and Doppler shift, respectively, of the target (if present), $\tau_{i}$ and $\nu_{i}$ are the delay and Doppler shift of the $i$ th scatterer, respectively, and $n(t)$ is additive noise. Note that the complex reflectivity of each scatterer fluctuates randomly with each pulse. This is due to the fact that small changes in range, on the order of the radar wavelength, may cause significant changes in the phase of the received signal [9]. In the scenario we consider, the interference due to clutter dominates the additive noise and we will henceforth neglect the latter. Also, we will focus on range estimation alone; the extension to include Doppler estimation is the subject of ongoing research. The received signal in (2) is sampled at a rate $f_{s}$ to yield a sequence $g^{k}[n]=g^{k}\left(n / f_{s}\right)$. This sampled signal is then matched filtered at each sampling instant to yield the sequence $r^{k}[n]$. We define $\mathbf{r}_{j} \in \mathbb{C}^{K \times 1}=\left[r^{0}[j], r^{1}[j], \ldots, r^{K-1}[j]\right]^{T}$ as the vector of matched-filtered outputs at the $j$ th delay or range bin. Then,

$$
\mathbf{r}_{j}=\sum_{n=-(N-1)}^{(N-1)} \mathbf{x}_{j+n} z_{s}[n],
$$

where $N$ is the length of the transmitted signal sequence $s[n]$, and $z_{s}[m]=\sum_{n=0}^{N-1} s[n] s^{*}[n-m],|m|<N$, is the value of the autocorrelation function of $s[n]$ at lag $m$.

\section{CLUTTER SUBSPACE ESTIMATION}

Next, we present the clutter subspace estimation algorithm. We also show that the subspace is waveform independent. 
Let $\Gamma_{j}$ define a neighborhood of range bins (with indices $j \pm n$, where $N \leq n<N+N_{Q}$ ) around the $j$ th bin for which we seek an estimate $\hat{Q}_{j}$ of the clutter subspace. The set $\Gamma_{j}$ thus includes $2 N_{Q}$ range bins whose returns do not include any contribution from the scatterers in bin $j$. We form the covariance matrix estimate

$$
\hat{R}_{j}=\frac{1}{2 N_{Q}} \sum_{n \in \Gamma_{j}} \mathbf{r}_{n} \mathbf{r}_{n}^{H},
$$

where the superscript $H$ denotes Hermitian transpose. Let $\hat{Q}_{j}$ denote the subspace spanned by the $N_{e}<K$ principal eigenvectors of $\hat{R}_{j}$. The remaining eigenvectors span a space $\hat{Q}_{j}^{\perp}$ and we define the orthogonal projection of the received signal on the clutter subspace as

$$
\mathbf{r}_{j}^{\perp}=\hat{Q}_{j}^{\perp^{H}} \mathbf{r}_{j},
$$

which constitute the clutter-suppressed signal.

From (3), the covariance $R_{j}$ of $\mathbf{r}_{j}$ may be written as

$$
\begin{aligned}
R_{j} & =E\left\{\mathbf{r}_{j} \mathbf{r}_{J}^{H}\right\} \\
& =\sum_{n=-(N-1)}^{N-1} \sum_{i=-(N-1)}^{N-1} E\left\{\mathbf{x}_{j+n} \mathbf{x}_{j+i}^{H}\right\} z_{s}[n] z_{s}^{*}[i],
\end{aligned}
$$

where $E\{\cdot\}$ is the expectation operator. From the CG model however, given the texture and covariance matrix $\Sigma, \mathbf{x}_{i}$ and $\mathbf{x}_{n}$ are independent if $i \neq n$. Also, $\mathbf{x}_{i}$ has zero mean and hence

$$
\begin{aligned}
R_{j} & =\sum_{n=-(N-1)}^{N-1} \mathcal{T}_{j+n} \boldsymbol{\Sigma}\left|z_{s}[n]\right|^{2} \\
& =\boldsymbol{\Sigma} \beta_{j},
\end{aligned}
$$

where the scalar $\beta_{j}$ is a function of the waveform. The eigenvectors of $R_{j}$ are identical to those of $\boldsymbol{\Sigma}$ and are thus waveform independent. The estimator of $\hat{R}_{j}$ requires only that the speckle covariance be constant across the training data and this is met by the CG model in which only the texture varies across range bins. The estimate $\hat{R}_{j}$ is thus a scaled estimate of $\Sigma$ and we conclude that the subspace estimates $\hat{Q}_{j}$ and $\hat{Q}_{j}^{\perp}$ are waveform independent.

\section{GLRT DETECTOR}

In this section, we develop the GLRT detector that operates on the clutter-suppressed data $\mathbf{r}_{j}^{\perp}$. An application of the Expectation-Maximization (EM) algorithm [10] to the estimation of the texture values and the speckle covariance matrix is also described.

With respect to a given range bin, we define the hypotheses $\mathbf{H}_{0}$ to indicate the presence of only clutter and $\mathbf{H}_{1}$ to indicate the presence of both clutter and target in that bin. We seek to form a detector based on the log-likelihood ratio test:

$$
\Lambda_{j}=\ln \frac{p\left(\mathbf{r}_{j}^{\perp} \mid \mathbf{H}_{1}\right)}{p\left(\mathbf{r}_{j}^{\perp} \mid \mathbf{H}_{0}\right)} \gtrless \gamma,
$$

where $\gamma$ is a threshold that is set to obtain a specified probability of false alarm. The probability density functions in (6) depend upon the distributions for the texture values $\mathcal{T}=$ $\mathcal{T}_{i}, i \in j+[-(N-1), N-1]$, the speckle covariance matrix $\Sigma$, and the variance of the target return $\sigma^{2} \mathbb{I}_{K}$. Thus,

$$
\Lambda_{j}=\ln \frac{E_{\left\{\mathcal{T}, \boldsymbol{\Sigma}, \sigma^{2}\right\}}\left\{p\left(\mathbf{r}_{j}^{\perp} \mid \mathbf{H}_{1}, \mathcal{T}, \boldsymbol{\Sigma}, \sigma^{2}\right)\right\}}{E_{\{\mathcal{T}, \boldsymbol{\Sigma}\}}\left\{p\left(\mathbf{r}_{j}^{\perp} \mid \mathbf{H}_{0}, \mathcal{T}, \boldsymbol{\Sigma}\right)\right\}} \gtrless \gamma,
$$

where $E_{\{a\}}\{\cdot\}$ indicates expectation conditioned on $a$. Since the distributions for the parameters in (7) are difficult to obtain, we instead form the GLRT by using estimates of the parameters so that

$$
\Lambda_{j}^{G L R T}=\ln \frac{p\left(\mathbf{r}_{j}^{\perp} \mid \mathbf{H}_{1}, \hat{\mathcal{T}}, \hat{\boldsymbol{\Sigma}}, \hat{\sigma}^{2}\right)}{p\left(\mathbf{r}_{j}^{\perp} \mid \mathbf{H}_{0}, \hat{\mathcal{T}}, \hat{\boldsymbol{\Sigma}}\right)} \gtrless \gamma^{\prime} .
$$

From (3) and (5), note that both the probability density functions in (8) are complex Gaussian. Once the parameters are estimated, these densities are known and the detection problem can be solved [11]. Next, we present the estimation of the texture and the speckle covariance matrix. Once these are obtained, the maximum likelihood estimate $\hat{\sigma}^{2}$ is obtained for a given value of $\mathbf{r}_{j}^{\perp}$.

\subsection{Texture and Speckle Covariance Estimation}

We seek the maximum-likelihood estimate of

$$
\boldsymbol{\theta}=\left\{\mathcal{T}_{j-(N-1)}, \ldots, \mathcal{T}_{j}, \ldots, \mathcal{T}_{j+(N-1)}, \boldsymbol{\Sigma}\right\}
$$

given the observed data

$$
\mathbf{r}=\left[\mathbf{r}_{j-(N-1)}^{H}, \ldots, \mathbf{r}_{j}^{H}, \ldots, \mathbf{r}_{j+(N-1)}^{H}\right]^{H} .
$$

However, from (3), the probability density of $\mathbf{r}$ depends on

$$
\Theta=\left\{\mathcal{T}_{j-2(N-1)}, \ldots, \mathcal{T}_{j}, \ldots, \mathcal{T}_{j+2(N-1)}, \boldsymbol{\Sigma}\right\}
$$

so that $\boldsymbol{\theta} \subset \Theta$. We will therefore seek

$$
\hat{\Theta}=\arg \max _{\Theta} p(\mathbf{r} ; \Theta),
$$

where $p(\mathbf{r} ; \Theta)$ is the probability density function of $\mathbf{r}$ that depends on $\Theta$. In (9), note that we treat the texture as a deterministic but unknown parameter rather than as a random process. This is in contrast to most clutter research, where a probability distribution is assigned to the texture and its parameters are estimated [12].

Since the maximization in (9) requires a complicated multidimensional search, we instead attempt to find an estimate of $\Theta$ that maximizes $p(\mathbf{x} ; \Theta)$, where

$$
\mathbf{x}=\left[\mathbf{x}_{j-2(N-1)}^{H}, \ldots, \mathbf{x}_{j}^{H}, \ldots, \mathbf{x}_{j+2(N-1)}^{H}\right]^{H}
$$


is the unobserved or complete data upon which the observed data $\mathbf{r}$ depends. Thus, we seek

$$
\hat{\Theta}=\arg \max _{\Theta} p(\mathbf{x} ; \Theta),
$$

which can be accomplished by the application of the EM algorithm as follows [10].

Starting with an initial guess $\hat{\Theta}^{(0)}$, let $\hat{\Theta}^{(l)}$ be the estimate of $\Theta$ after $l$ iterations. Then the EM algorithm is given by

- E (expectation) step: Compute

$$
U\left(\Theta, \hat{\Theta}^{(l)}\right)=E\left\{\ln p(\mathbf{x} ; \Theta) \mid \mathbf{r}, \hat{\Theta}^{(l)}\right\}
$$

- $\mathbf{M}$ (maximization) step: Obtain

$$
\hat{\Theta}^{(l+1)}=\arg \max _{\Theta} U\left(\Theta, \hat{\Theta}^{(l)}\right) .
$$

The details of the computations in (10) and (11) follow [13], and are not be provided here. Making use of the Kronecker product for simplification, we can show that the final results are obtained as a simultaneous solution of

$$
\begin{aligned}
\hat{\mathcal{T}}_{i} & =\frac{\operatorname{tr}\left\{\hat{\boldsymbol{\Sigma}}^{-1} \Lambda_{i}\right\}}{K}, \quad i \in j+[-2(N-1), 2(N-1)] \\
\hat{\boldsymbol{\Sigma}} & =\frac{1}{4 N-3} \sum_{i=j-2(N-1)}^{j+2(N-1)} \frac{\Lambda_{i}}{\hat{\mathcal{T}}_{i}}
\end{aligned}
$$

where $\Lambda_{i}$ is the $i$ th $K \times K$ matrix on the diagonal of

$$
\widehat{\mathbf{x x}}^{H}=E\left\{\mathbf{x} \mathbf{x}^{H} \mid \mathbf{r} ; \hat{\Theta}^{(l)}\right\} .
$$

Note that the estimate of texture in the range bin that contains the target is likely to be incorrect and cannot be used as an estimate of the texture statistics under the $\mathbf{H}_{0}$ hypothesis. It is therefore necessary to obtain this estimate when the return in that bin is not influenced by the target. Assuming that a moving target is located in range bin $j$ at dwell $k$, we use the estimate of $\mathcal{T}_{j}$ from dwell $k-1$ as the required $\mathbf{H}_{0}$ statistic for this bin at dwell $k$. Note that, this procedure is sub-optimal because the texture will undergo changes between dwells. However, from the correlation properties of the texture, we can expect these changes to be small if the interdwell duration is on the order of 0.5-1 s. In practice, this approach works reasonably well.

\section{SIMULATIONS}

Our simulation study consists of the detection of a moving target in the presence of K-distributed sea clutter, which specifies a gamma distribution for the texture. Note that the development in Sections 4 and 5 does not depend on any specific probability distribution for the texture. The generation of correlated $\mathrm{K}$-distributed sea clutter is not trivial and has received

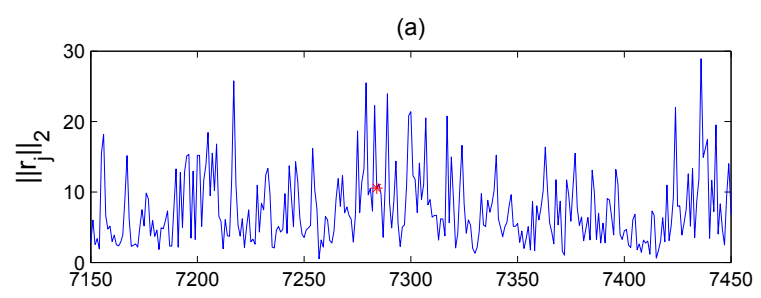

(b)

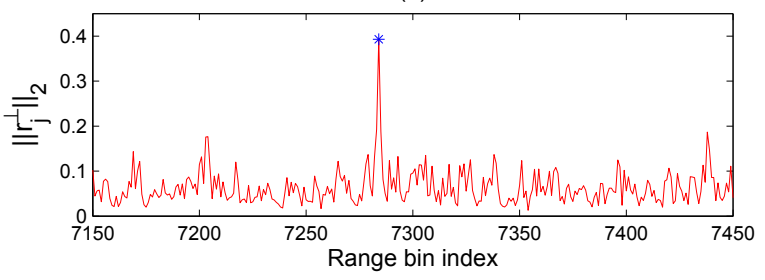

Fig. 3. Norm of (a) the matched-filtered output $\left\|\mathbf{r}_{j}\right\|_{2}$, and (b) the clutter-suppressed output $\left\|\mathbf{r}_{j}^{\perp}\right\|_{2}$, for a typical dwell with $\mathrm{SCR}=-35 \mathrm{~dB}$. The true target range bin is marked with an asterisk.

significant attention in the literature (see [14] for a review). Our method generates the complex reflectivity $\mathbf{x}_{i}=\sqrt{\mathcal{T}_{i}} \mathcal{X}_{i}$, where the speckle variable $\mathcal{X}_{i}$ is drawn from a complex Gaussian distribution with zero mean and covariance matrix $\boldsymbol{\Sigma}$, derived from the autocorrelation function shown in Fig. 1. The texture variables $\mathcal{T}_{i}$ are drawn from a gamma distribution and correlated in time according to the autocorrelation function shown in Fig. 2 using a memoryless nonlinear transformation [15]. Briefly, this method generates a gamma distributed variate $\mathcal{T}_{i}$ from the Gaussian variable $y_{i}$ as the solution of

$$
\int_{\mathcal{T}_{i}}^{\infty} \frac{2 b^{2 \nu}}{\Gamma(\nu)} u^{2 \nu-1} \mathrm{e}^{-b^{2} u^{2}} d u=\frac{1}{\sqrt{2 \pi}} \int_{y_{i}}^{\infty} \mathrm{e}^{-\frac{v^{2}}{2}} d v .
$$

The correlation properties of $\mathcal{T}_{i}$ can be expressed in terms of the correlation properties of $y_{i}$ [15]. This mapping can then be inverted to provide a means of generating $\mathcal{T}_{i}$ with the desired correlation. In our simulations, we use $b=1$ and $\nu=0.5$ in (12), which results in highly non-Gaussian clutter.

We assume a PRF of $10 \mathrm{kHz}$ and transmit $K=10$ pulses of a $1.5 \mu$ s duration linear frequency modulated (LFM) chirp with a frequency sweep of $100 \mathrm{MHz}$. The specular reflections are thus correlated within a sub-dwell. We use a neighborhood of $N_{Q}=20$ range bins in the estimation of the clutter covariance in (4), and the eigen vectors in the subspace $\hat{Q}_{j}^{\perp}$ correspond to the smallest eigen values that contribute $0.01 \%$ of the total energy. The amplitude of the target return was sampled from a zero mean complex Gaussian process with covariance matrix $\sigma^{2} \mathbb{I}_{K}$, where $\sigma^{2}$ was chosen to satisfy specified values of SCR. We define the SCR to be the ratio of the target signal power to the total power of the clutter in the range bin containing the target.

A typical example of the clutter suppression behavior is 


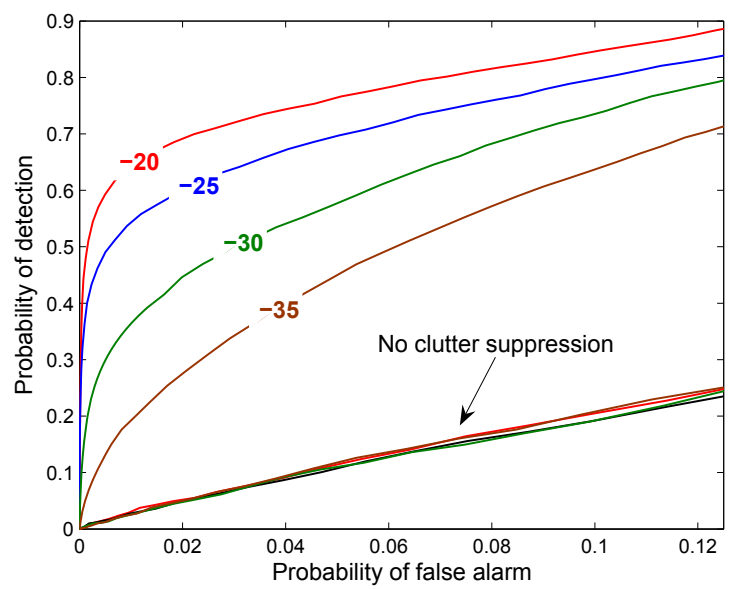

Fig. 4. ROC curves with and without clutter suppression using a static LFM chirp waveform. The indicated values correspond to SCR in $\mathrm{dB}$.

presented in Fig. 3, where the $L_{2}$ norm of the cluttersuppressed signal $\mathbf{r}_{j}^{\perp}$ and the raw matched-filtered signal (without clutter suppression) $\mathbf{r}_{j}$ are shown. Fig. 4 demonstrates the receiver operating characteristic (ROC) curves for the GLRT detector operating on the clutter-suppressed signal for a number of different SCR values. The performance curves for detection based on the matched-filtered output are also shown for comparison. We note that the clutter suppression algorithm significantly improves detection performance.

\section{CONCLUSIONS}

The problem of detecting small targets on the ocean surface in heavy sea clutter remains challenging. However, a great deal is known about the statistical properties of sea clutter echoes, and this may be exploited to improve detection performance. We have demonstrated an online, subspace-based approach to sea clutter mitigation. We used the CG model to build a GLRT detector that uses estimates of the texture provided by the EM algorithm. Simulation results were shown to demonstrate the improvement in detection performance.

The estimate of the texture obtained in Section 5 can be used to design the next transmitted waveform. Note that the matched-filtering operation smears energy from one range bin to another leading to out-of-bin clutter contributions in the range bin of interest. This smearing depends upon the autocorrelation function of the waveform and can be minimized by designing it to be small where the clutter is strong [16].

\section{REFERENCES}

[1] D. F. DeLong and E. M. Hofstetter, "On the design of optimum radar waveforms for clutter rejection," IEEE Trans. Inform. Theory, vol. IT-13, no. 3, pp. 454-463, Jul. 1967.
[2] S. Haykin, R. Bakker, and B. W. Currie, "Uncovering nonlinear dynamics - The case study of sea clutter," Proc. IEEE, vol. 90, pp. 860-881, May 2002.

[3] C. J. Oliver, "Correlated K-distributed clutter models," Optica Acta, vol. 32, no. 12, pp. 1515-1547, 1985.

[4] A. Farina, F. Gini, M. V. Greco, and L. Verrazzani, "High resolution sea clutter data: Statistical analysis of recorded live data," IEE Proc. on Radar, Sonar and Navigation, vol. 144, no. 3, pp. 121-130, Jun. 1997.

[5] K.D. Ward, C.J. Baker, and S. Watts, "Maritime surveillance radar Part I : Radar scattering from the ocean surface," IEE Proc. F: Communications, Radar and Sig. Proc., vol. 137, no. 2, pp. 51-62, Apr. 1990.

[6] S. Suvorova, B. Moran, and M. Viola, "Adaptive modeling of sea clutter and detection of small targets in heavy sea clutter," IEEE International Radar Conference, pp. 614-618, 2003.

[7] K. J. Sangston and K. R. Gerlach, "Coherent detection of radar targets in a non-Gaussian background," IEEE Trans. Aerosp. Electron. Syst., vol. 30, no. 2, pp. 330-340, Apr. 1994.

[8] A. Drosopoulos, "Description of the OHGR database," Tech. Rep. 94-14, Defence Research Establishment, Ottawa, Dec. 1994.

[9] B. Friedlander, "A subspace framework for adaptive radar waveform design," Asilomar Conf. on Signals, Systems and Computers, pp. 1135-1195, Oct. 2005.

[10] A. Dempster, N. Laird, and D. Rubin, "Maximum likelihood estimation from incomplete data via the EM algorithm," Journal of the Royal Statistical Society, Series B, vol. 39, no. 1, pp. 1-38, 1977.

[11] S. M. Kay, Fundamentals of Statistical Signal Processing: Detection Theory, vol. 2, Prentice-Hall, Englewood Cliffs, N.J., 1993.

[12] J. Wang, A. Dogandžic, and A. Nehorai, "Maximum likelihood estimation of compound-Gaussian clutter and target parameters," IEEE Trans. Sig. Proc., vol. 54, pp. 3884-3898, Oct. 2006.

[13] M. Feder and E. Weinstein, "Parameter estimation of superimposed signals using the EM algorithm," IEEE Trans. Acoust., Speech, Signal Processing, vol. 36, no. 4, pp. 477-489, Apr. 1988.

[14] I. Antipov, "Simulation of sea clutter returns," Tech. Rep. DSTO-TR-0679, Defence Science and Technology Organisation, Salisbury, South Australia, Jun. 1998.

[15] R. J. A. Tough and K. D. Ward, "The correlation properties of gamma and other non-Gaussian processes generated by memoryless nonlinear transformation," J. Phys. D: Appl. Phys., vol. 32, pp. 3075-3084, 1999.

[16] S. P. Sira, A. Papandreou-Suppappola, D. Morrell, D. Cochran, W. Moran, S. Howard, and R. Calderbank, "Adaptive waveform design for improved detection of low-RCS targets in heavy sea clutter," IEEE Journal on Special Topics in Signal Processing: Special Issue on Adaptive Waveform Design for Agile Sensing and Communication, Jun. 2007, In review. 